\title{
Surface scanning for 3D dose calculation in intraoperative electron radiation therapy
}

Verónica García-Vázquez ${ }^{1 *}$ (i), Begoña Sesé-Lucio1, Felipe A. Calvo ${ }^{1,2,3,4}$, Juan J. Vaquero ${ }^{5,1}$, Manuel Desco ${ }^{5,1,6,7}$ and Javier Pascau ${ }^{5,1,6}$

\begin{abstract}
Background: Dose calculations in intraoperative electron radiation therapy (IOERT) rely on the conventional assumption of water-equivalent tissues at the applicator end, which defines a flat irradiation surface. However, the shape of the irradiation surface modifies the dose distribution. Our study explores, for the first time, the use of surface scanning methods for three-dimensional dose calculation of IOERT.

Methods: Two different three-dimensional scanning technologies were evaluated in a simulated IOERT scenario: a tracked conoscopic holography sensor (ConoProbe) and a structured-light three-dimensional scanner (Artec). Dose distributions obtained from computed tomography studies of the surgical field (gold standard) were compared with those calculated under the conventional assumption or from pseudocomputed tomography studies based on surfaces.

Results: In the simulated IOERT scenario, the conventional assumption led to an average gamma pass rate of 39.9\% for dose values greater than 10\% (two configurations, with and without blood in the surgical field). Results improved when considering surfaces in the dose calculation (88.5\% for ConoProbe and $92.9 \%$ for Artec).

Conclusions: More accurate three-dimensional dose distributions were obtained when considering surfaces in the dose calculation of the simulated surgical field. The structured-light three-dimensional scanner provided the best results in terms of dose distributions. The findings obtained in this specific experimental setup warrant further research on surface scanning in the IOERT context owing to the clinical interest of improving the documentation of the actual IOERT scenario.
\end{abstract}

Keywords: IOERT, Intraoperative radiotherapy, Surface scanning, Conoscopic holography, Structured-light 3D scanner, Dose distribution

\section{Background}

Intraoperative electron radiation therapy (IOERT) refers to the delivery of a single-fraction, high-energy electron beam during surgery with the goal of promoting local cancer control [1-3]. The target volume (namely a post-resection tumour bed or the macroscopic residue after partial resection) is irradiated by using a specific applicator that pushes aside healthy tissues and collimates the electron beam generated by a linear accelerator (LINAC) $[1,4,5]$.

\footnotetext{
* Correspondence: vgarcia@hggm.es

${ }^{1}$ Instituto de Investigación Sanitaria Gregorio Marañón. Calle Doctor

Esquerdo, 46, 28007, Madrid, Spain

Full list of author information is available at the end of the article
}

IOERT dosimetry relies on the conventional assumption of water-equivalent tissues in both stopping and scattering power at the applicator end, which defines a flat irradiation surface. Previous literature reported small differences between prescribed doses under previous assumption and measured doses using in-vivo dosimetry in the case of breast IOERT scenarios $[6,7]$. However, Costa et al. [8], by placing radiochromic films on irradiation surfaces, showed that clinical two-dimensional (2D) dose distributions in pelvic IOERT scenarios frequently differ from the expected ones. In a previous study, Costa et al. [9] simulated these IOERT scenarios with solid water slabs and a radiotherapy bolus, highlighting that the shape 
of the irradiation surface (namely step-like or curved surfaces) modified the dose distribution. Documentation of the actual scenario is relevant to the quality assurance of IOERT and the proper assessment of clinical results. However, the applicator position, the angle of beam incidence in relation to the patient's anatomy and the shape of the irradiation surface are not available in IOERT records. Intraoperative computed tomography (CT) studies with the applicator placed on the tumour bed would allow the calculation of three-dimensional (3D) dose distributions of the actual scenario before irradiation since these images include tissue heterogeneities, surface irregularities of the irradiated volume and the air gap from the applicator end to the tumour bed. A portable CT scanner inside the operating room or a LINAC with on-board $\mathrm{kV}$ cone beam CT may be used for that purpose [10]. However, a low number of IOERT interventions per week may not support the installation costs of in-room imaging devices [11]. In addition, intraoperative CT studies with metal artefacts (for example, owing to shielding discs and surgical retractors) cannot be directly used for dose calculation since CT values are substantially altered.

An intermediate approach between the IOERT conventional assumption and the use of intraoperative CT images would involve 3D scanning the surface of the tumour bed and the inclusion of these data in the dose calculation. In a preliminary study [12], the authors evaluated four different technologies to scan surfaces, selecting a custom-made structured-light 3D scanner as the most appropriate for IOERT in terms of resolution, accuracy, acquisition time and cost. However, this statement was based on scanning a half body mannequin, not simulating an actual IOERT scenario and calculating dose distributions. Brudfors et al. [13] presented an open-source software for scanning surfaces with a tracked conoscopic holography sensor that could also be used in IOERT. This system has been previously evaluated for clinical usage (for example, to scan human cadaver kidneys $[14,15]$ and resection cavities during neurosurgeries [16]).

In this study we aimed to explore, for the first time, the use of surface scanning methods for 3D dose calculation in IOERT. Two different 3D scanning technologies were evaluated in a simulated IOERT scenario, a tracked conoscopic holography sensor and a structured-light 3D scanner.

\section{Methods}

In this section, we describe the 3D scanning systems assessed (section "3D scanning systems"), the process of creating 3D images from surfaces (section "Surface to pseudo-CT study for IOERT dose calculation") and the experiment designed to evaluate these non-contact devices for 3D dose calculation in IOERT (section "Experiment"). A simulated surgical field was scanned with both 3D scanning systems. 3D dose distributions obtained from CT studies of the surgical field (gold standard) were compared with those calculated under the conventional assumption of waterequivalent tissues at the applicator end and with those calculated from pseudo-CT studies based on surfaces.

\section{D scanning systems}

\section{Tracked conoscopic holography sensor}

A conoscopic holography sensor measures distances to objects with an interferometric technique based on the double refraction of uniaxial crystals [17]. The collinearity between the laser beam emitted and the cone of light returned from the scanned object enables measurements of sharp edges and inside narrow cavities. Distances are converted into $3 \mathrm{D}$ coordinates of the scanned surface by tracking the sensor [14].

The configuration selected in this study was that presented in [13]: a ConoProbe Mark 10 sensor (Optimet, Optical Metrology Ltd) (working distance from $155 \mathrm{~mm}$ to $336 \mathrm{~mm}$ with the $250-\mathrm{mm}$ objective lens as the origin, laser wavelength $655 \mathrm{~nm}$, laser spot size $107 \mu \mathrm{m}$, laser power lower than $1 \mathrm{~mW}$ and weight $0.72 \mathrm{~kg}$ [18]) was tracked by a multi-camera optical tracking system OptiTrack (NaturalPoint, Inc.) with three optical cameras FLEX:V100R2 (data rate $50 \mathrm{~Hz}$ ).

The data acquired with the tracked ConoProbe sensor (abbreviated hereafter to "ConoProbe") is an unorganised point set that is converted to a triangle mesh by calculating its oriented surface normals and applying Poisson surface reconstruction [19] by means of open-source software MeshLab [20].

\section{Structured-light 3D scanner}

This 3D scanning technology is based on an incoherent light source that projects structured 2D patterns onto the scanned object, and a camera at a different viewpoint that records the patterns geometrically distorted by the object surface.

The specifications of the handheld 3D scanner selected in this study, Artec Eva (abbreviated hereafter to "Artec"), are: 3D resolution up to $0.5 \mathrm{~mm}$, working distance from $0.4 \mathrm{~m}$ to $1 \mathrm{~m}$, frame rate up to $16 \mathrm{fps}$ and weight $0.85 \mathrm{~kg}$ [21]. This solution includes another camera to capture colour texture. The software Artec Studio 10 manages the capturing process, data post-processing, 3D model (triangle mesh) creation and texture mapping.

\section{Surface to pseudo-CT study for IOERT dose calculation}

Dose distributions are calculated with a treatment planning system (TPS) specifically developed for IOERT (radiance, GMV) [22, 23] based on a Monte Carlo algorithm that takes account of the LINAC 
phase space [24] and tissue heterogeneities [25] (data obtained from CT studies after converting Hounsfield units (HU) values to physical density $[10,25])$.

3D surfaces are converted into pseudo-CT studies for dose calculation. In this process (Fig. 1), we first generate a watertight surface by extruding a contour close to the mesh edge (out of the surgical field) and covering the extra walls generated with a lid (Autodesk software Meshmixer [26] and Artec Studio 10). Second, the watertight surface is converted to a $3 \mathrm{D}$ binary image by applying a ray intersection method [27] similar to that presented in [28]. Then, this 3D image is transformed into a pseudo-CT study (defined as a 3D image not acquired in a CT scanner but with values in the HU scale) by setting the voxels above the scanned surface to the CT value of air $(-1000 \mathrm{HU})$ and the remaining voxels to water value $(0 \mathrm{HU})$.

\section{Experiment}

\section{Experiment set-up}

The experiment was carried out in a CT simulator room in order to acquire reference $\mathrm{CT}$ studies. The cameras of the optical tracking system were statically attached to three STOLMEN posts (Inter IKEA Systems B.V.) around the table of an Aquilion ${ }^{\text {Tx }}$ Large Bore CT simulator (Toshiba) (Fig. 2).

A surgical field was simulated with a large piece of beef (weight $6 \mathrm{~kg}$ ) placed at the bottom of a plastic box (volume $28 \times 39 \times 57 \mathrm{~cm}$ ), on the CT table, as shown in
Fig. 2. This large container simulated the volume of a sterile field with surgical retractors that may hinder 3D scanning. Six metallic nipple markers (diameter $1 \mathrm{~mm}$, SL-10, The Suremark Company) were placed on cuboids of different heights around the phantom (Fig. 2). These markers were used as landmarks for mapping surfaces and CT studies into a common coordinate space.

\section{Acquisition protocol}

The details of the acquisition protocol are as follows:

1. A CT study of the surgical field and the markers (CTpreSurf or CT study acquired before surface scanning) was acquired with the following parameters: voltage $120 \mathrm{kVp}$, exposure $37 \pm 10 \mathrm{mAs}$ (mean \pm standard deviation), matrix size $512 \times$ $512 \times 961$ and voxel size $0.729 \times 0.729 \times 0.500 \mathrm{~mm}$.

2. Laser beam (ConoProbe) sweeping of the surgical field and the markers (Fig. 2, top right) (minimum signal-to-noise ratio set to $40 \%$ ).

3. Surface scanning of the surgical field and the markers (Fig. 2, top right) with Artec.

Steps 2 and 3 were carried out from only one viewpoint to simulate the difficulty of moving around the patient in the operating room with our wired 3D scanners connected to both the power supply and the computer.
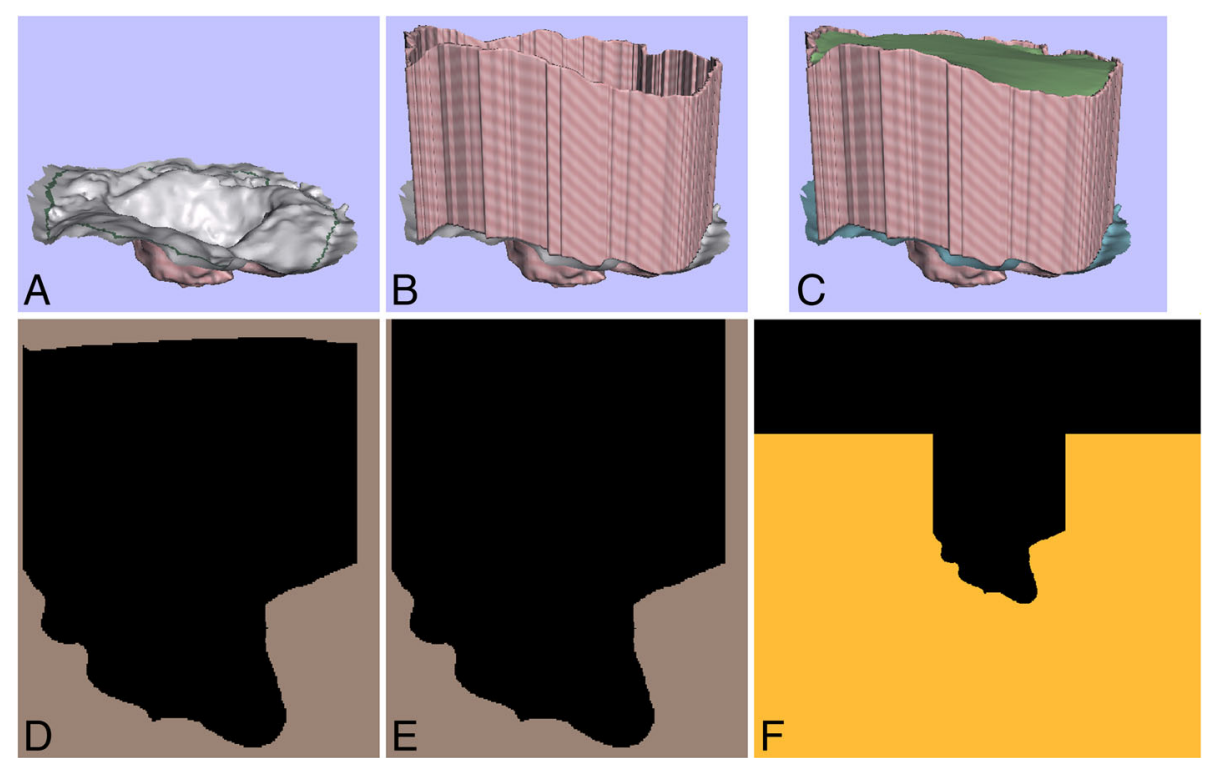

Fig. 1 Conversion from 3D surface into pseudo-CT study. (a) 3D surface with a green contour delineated close to the mesh edge. (b) Extra walls generated after extruding the contour close to the mesh edge. (c) Green lid covering those extra walls to obtain a watertight surface. (d) Axial view of the $3 \mathrm{D}$ binary image generated after applying the ray intersection method to the watertight surface. The matrix size of this $3 \mathrm{D}$ image is limited to the boundaries of the watertight surface. (e) Axial view of the 3D binary image after correcting the region above the lid. (f) Axial view of the pseudo-CT study obtained by increasing the matrix size of the 3D binary image, and then by setting the black voxels to the CT value of air and the remaining voxels to the CT value of water (orange colour in the pseudo-CT study) 

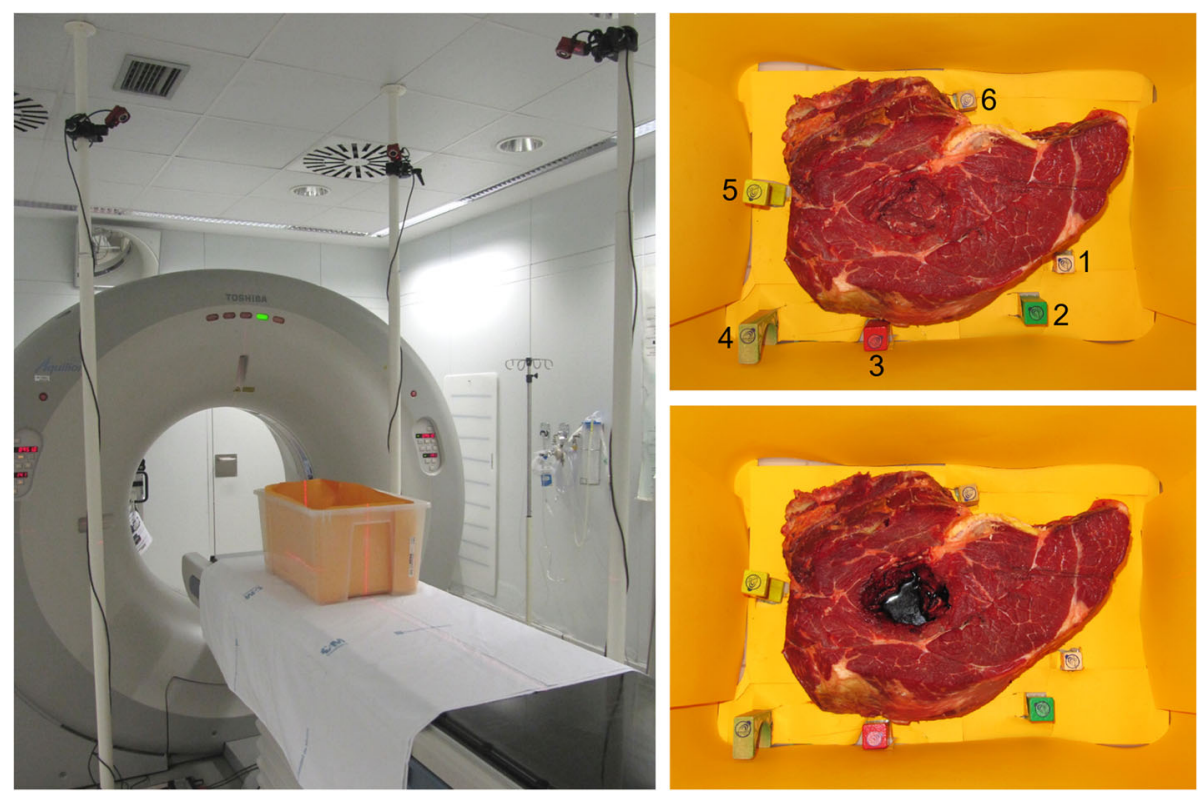

Fig. 2 Experiment set-up. On the left, the CT simulator room with the multi-camera optical tracking system and the plastic box, which contained the simulated surgical field and the markers, on the $\mathrm{CT}$ table. The three optical cameras of the tracking system were attached to three posts. On the right, the simulated surgical field and the six markers placed at different heights. The configuration without blood is shown at the top right while the configuration with blood is depicted at the bottom right

4. Step 1 was repeated with the same initial position of the patient table to obtain CTpostSurf (CT study acquired after surface scanning), which was used to check the stability of the whole setting (surgical field and markers) by comparing CTpostSurf and CTpreSurf. This verification was done because the surface irregularities of the simulated surgical field and the position of the markers could vary in steps 2 and 3 from the original setting shown in CTpreSurf due to the force of gravity, the movements of the CT table after CTpreSurf acquisition or a wrong attachment of the markers. The comparison was performed by superimposing CTpostSurf to CTpreSurf and calculating the rootmean-square error [RMSE] between the intensities from both studies without applying any alignment.

5. Steps 1 through 4 were repeated after adding $40 \mathrm{ml}$ of pig blood with heparin (anticoagulant) to the surgical field (Fig. 2, bottom right) in order to simulate accumulation of biological fluid before irradiation.

\section{Surface registration}

Scanned surfaces were mapped to CTpreSurf to set a common coordinate space for comparisons in each configuration (with and without blood). The transformation matrix was calculated with a landmark rigid registration algorithm based on singular value decomposition [29] and the 3D coordinates of each marker (its centroid in the CT study and a point on its 3D surface) using 3D Slicer software [30] and SlicerIGT extension [31]. Colour texture (visible light picture data from the surface) was essential for obtaining the marker location in the Artec surfaces since the markers were not properly identified in the triangle meshes.

\section{IOERT dose distributions}

Registered surfaces were converted to pseudo-CT studies of 1-mm isotropic voxel size by applying the method described in section "Surface to pseudo-CT study for IOERT dose calculation" (obtaining CTConoProbe and CTArtec for ConoProbe and Artec surfaces respectively). CTpreSurf studies were also resampled to that voxel size.

An IOERT case was simulated in the TPS by placing a virtual applicator on CTpreSurf, CTConoProbe and CTArtec (parameters: applicator diameter $70 \mathrm{~mm}$, bevel angle $30^{\circ}$ and electron energy $9 \mathrm{MeV}$ ). The following 3D dose distributions were calculated for each configuration (with and without blood) with a Monte Carlo algorithm (error tolerance 1\% [parameter that limits the maximum estimated uncertainty of the simulated dose for doses higher than $50 \%$ of the maximum dose] and resolution $1.0 \mathrm{~mm}$ ) and the phase space of a conventional LINAC (Varian 21EX):

- D_CTpreSurf (considered as the gold standard) was calculated with CTpreSurf. 
- D_water was calculated with CTpreSurf after selecting Water option in the TPS to obtain the dose distribution under the conventional assumption of water-equivalent tissues at the applicator end. $D \_$water was obtained to assess the dose deviation when not including tissue heterogeneities, surface irregularities of the irradiated volume and the air gap from the applicator end to the tumour bed in the dose calculation.

- D_CTtissue\&air was calculated after converting CTpreSurf to another pseudo-CT study (CTtissue\&air) that had only two different $\mathrm{CT}$ values (namely water [0 HU] and air [- $1000 \mathrm{HU}])$. D_CTtissue\&air was used to assess the dose deviation when applying the same simplification of CTConoProbe and CTArtec in the dose calculation (namely not taking account of tissue heterogeneities but including surface irregularities of the irradiated volume and the air gap from the applicator end to the tumour bed). CTtissue\&air was obtained by first segmenting the air in CTpreSurf using intensity thresholding method (maximum limit - $500 \mathrm{HU}$ ), and then setting those voxels to the CT value of air and the remaining voxels (specifically, tissue) to the $\mathrm{CT}$ value of water.

- D_CTConoProbe and D_CTArtec were calculated with CTConoProbe and CTArtec respectively.

The output of the Monte Carlo algorithm was a 3D dose distribution in percentage where $100 \%$ corresponded to the maximum dose along the clinical axis measured in a water phantom for the selected applicator diameter and energy of the electron beam (in this study, $70 \mathrm{~mm}$ and $9 \mathrm{MeV}$ respectively), the bevel angle $0^{\circ}$, and the source-to-surface distance set when conducting the measurements for modelling the LINAC.

D_water, D_CTtissue\&air, D_CTConoProbe and D_CTArtec were compared with D_CTpreSurf using global normalisation [32], and a 3D gamma criteria of $3 \% / 3 \mathrm{~mm}$ (thresholds accepted clinically) for dose values greater than $10 \%$ or $70 \%$ (to focus on high-dose regions) [10]. 3D gamma analyses did not take account of dose differences in voxels not belonging to tissue in CTpreSurf.

\section{Evaluation of "Surface to pseudo-CT study" conversion process}

A source of error when using surface scanning methods for 3D IOERT dose calculation is the conversion from a $3 \mathrm{D}$ surface into a pseudo-CT study. The surface created to assess this process was reproSurf, which was created by first segmenting the voxels with the CT value of water in CTtissue\&air (configuration without blood), then generating a watertight surface (both steps done with 3D Slicer software), and finally selecting a non-manifold surface of the surgical field like that obtained with ConoProbe and Artec (step done with Meshmixer software). The configuration chosen was that without blood since its surface was more irregular than that of the configuration with blood. A pseudo-CT study (CTreproSurf) was generated by applying the method detailed in section "Surface to pseudo-CT study for IOERT dose calculation" to reproSurf.

The evaluation consisted in comparing the 3D dose distribution calculated with CTreproSurf (D_CTreproSurf) and the reference 3D dose distribution D_CTtissue\&air, which was calculated with the CT study used to generate reproSurf. CTreproSurf included both the error of generating the surface from CTtissue\&air and the error when converting that surface into a pseudo-CT study. D_CTreproSurf was obtained with the same IOERT parameters detailed in section "IOERT dose distributions" and both dose distributions were compared using the same method described in section "IOERT dose distributions". This comparison enabled us to assess the influence of the conversion from a 3D surface into a pseudo-CT study on the dose calculation.

Table 1 summarises the image studies and 3D dose distributions previously explained in sections "Acquisition protocol", "IOERT dose distributions" and "Evaluation of 'Surface to pseudo-CT study' conversion process". Throughout the article, the prefix "CT" refers to the CT/pseudo-CT study and the prefix "D_CT" refers to the $3 \mathrm{D}$ dose distribution calculated from the CT/pseudo-CT study.

Table 1 Summary of CT/pseudo-CT studies and 3D dose distributions obtained in the experiment

\begin{tabular}{|c|c|c|c|c|c|}
\hline & $\begin{array}{l}\text { CT acquisition before } \\
\text { surface scanning }^{\mathrm{a}}\end{array}$ & $\begin{array}{l}\text { Surface scan with } \\
\text { Conoprobe }^{a}\end{array}$ & $\begin{array}{l}\text { Surface scan } \\
\text { with Artec }^{a}\end{array}$ & $\begin{array}{l}\text { CT acquisition after } \\
\text { surface scanning }\end{array}$ & $\begin{array}{l}\text { Surface from } \\
\text { CTpreSurf }^{\mathrm{b}}\end{array}$ \\
\hline Generated 3D images & $\begin{array}{l}\text { CTpresurf } \\
\text { CTtissue\&air }\end{array}$ & CTConoProbe & CTArtec & CTpostSurf & CTreprosurf \\
\hline 3D dose distributions & $\begin{array}{l}\text { D_CTpresurf } \\
\text { D_water } \\
\text { D_CTtissue\&air }\end{array}$ & D_CTConoProbe & D_CTArtec & & D_CTreprosurf \\
\hline
\end{tabular}

acquisition protocol detailed in section "Acquisition protocol"

bStep detailed in section "Evaluation of 'Surface to pseudo-CT study' conversion process" 


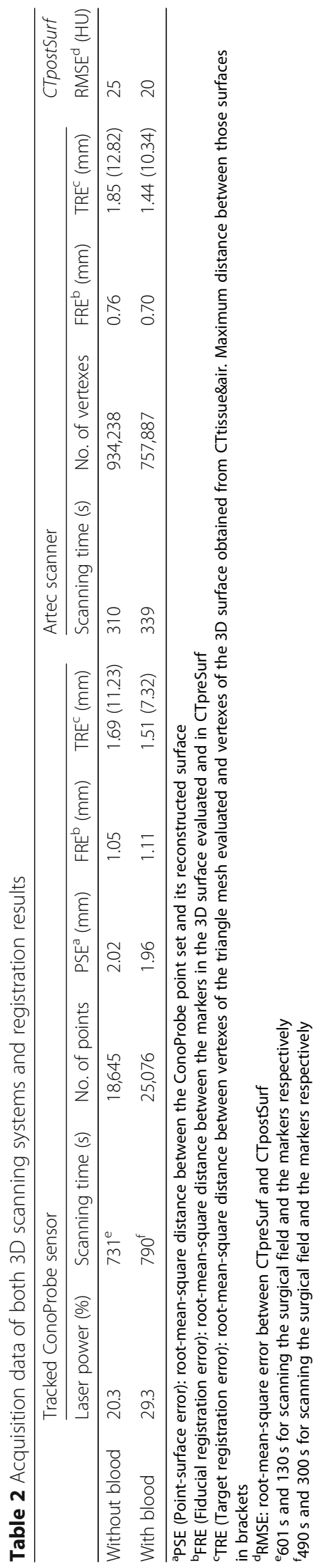




\section{Results}

Table 2 summarises the details of the surface scanning with both devices. The laser power (ConoProbe) was manually increased in the configuration with blood owing to the absorption of the red laser light. The scanning process of both the surgical field and the markers with ConoProbe took longer than with Artec, although the number of vertexes acquired with the latter was higher. Additional post-processing of Artec data (namely removal of noisy data, selection of surgical field and hole closing) was more laborious than that for ConoProbe data (namely outlier removal and selection of surgical field). The point-surface error (PSE, root-mean-square distance between the ConoProbe point set and its reconstructed surface) was around $2 \mathrm{~mm}$ (Table 2). This difference was not calculated for Artec since its output was a triangle mesh, not a point set.

In each configuration, CTpostSurf was superimposed to CTpreSurf to check the stability of the whole setting (surgical field and markers). Table 2 shows the RMSE between both studies without applying any alignment. The RMSE was lower than $26 \mathrm{HU}$ in both configurations. In addition, the only difference found when comparing CTpreSurf in both configurations was the blood at the base of the surgical field. The position and orientation difference between the four CT studies was neglectable (lower than $0.30 \mathrm{~mm}$ and $0.05^{\circ}$ respectively). These values were obtained from the transformation matrix calculated when mapping CTpreSurf (configuration with blood) and CTpostSurf (both configurations) to CTpreSurf (configuration without blood) with the landmark rigid registration algorithm explained in section "Surface registration" and the $3 \mathrm{D}$ coordinates of the markers.

Table 2 shows the registration results between 3D surfaces and CTpreSurf. Target registration error (TRE, root-mean-square distance between vertexes of each triangle mesh and vertexes of the 3D surface obtained from CTtissue\&air) was lower than $1.9 \mathrm{~mm}$ in all cases. Some holes inside the surgical field did not perfectly match when comparing CTtissue\&air, and the pseudo-CT studies CTConoProbe and CTArtec, as depicted in Fig. 3.

Figure 4 illustrates the complexity of the simulated tumour bed and the IOERT scenario set in each configuration. The maximum distance from the applicator end to the surface of the simulated tumour bed was $33.5 \mathrm{~mm}$ (configuration without blood) and $13.2 \mathrm{~mm}$ (configuration with blood). Dose distributions are shown in Fig. 5 and gamma pass rates are detailed in Table 3. D_CTtissue\&air closely followed D_CTpreSurf in both configurations (average gamma pass rate $99.7 \%$ for dose values greater than 10\%) while D_water differed from D_CTpreSurf (39.9\%). A better dose agreement was found in the case of D_CTConoProbe (88.5\%) and D_CTArtec (92.9\%). Figure 6 shows the gamma distribution for the dose distributions evaluated to identify, in that axial view, where the gamma criteria failed in the comparison for dose values greater than $10 \%$.

Figure 7 shows the agreement between the pseudo-CT studies CTreproSurf and CTtissue\&air. These studies were used to assess the influence of the conversion from a $3 \mathrm{D}$ surface into a pseudo-CT study on the dose calculation. The percentage of voxels fulfilling the 3D gamma criteria when comparing D_CTreproSurf with D_CTtissue\&air was $100.0 \%$ for dose values greater than $10 \%$ and for dose values greater than $70 \%$.

\section{Discussion}

Two different 3D scanning technologies were evaluated in the context of 3D dose calculation in IOERT. Both devices can scan at a distance greater than $30 \mathrm{~cm}$ (minimum safety margin between unsterile personnel and the sterile field [33]), suitable for the IOERT theatre. Advantages of Artec over ConoProbe are a larger working distance (up to $1 \mathrm{~m}$ ) and a shorter scanning time. Nevertheless, ConoProbe can obtain better measurements inside narrow cavities since its laser beam can access them more easily than the structured 2D patterns generated by the Artec projector. However, neither device could completely scan the deeper hole of the simulated surgical field (Fig. 3, red arrows), although ConoProbe was slightly better in this task.

CT studies (namely CTpreSurf and CTpostSurf) were used to check the stability of the whole setting (surgical field and markers) and to obtain reference surfaces/studies and dose distributions. The results of the comparison between CTpreSurf and CTpostSurf showed a similar scenario in each configuration (with and without blood). In addition, a similar IOERT case was simulated in both configurations except for blood accumulation. The air gap of the configuration without blood (maximum distance from the applicator end to the surface of the simulated tumour bed $33.5 \mathrm{~mm}$ ) was within the range seen in limb sarcomas (air gaps up to $5 \mathrm{~cm}$ [34]). However, the IOERT scenario simulated is not realistic for breast IOERT, where the smoothness of the irradiation surface and a minimum air gap from the applicator end to the tumour bed can be ensured by other means [35].

The use of surface scanning for 3D dose calculation in IOERT is based on assuming water-equivalent tissues at the surface of the tumour bed, not at the applicator end as in the conventional assumption. The gamma pass rates obtained with D_CTtissue\&air validated this assumption for our experiment (average value 99.7\% for dose values greater than 10\%) and therefore lower gamma pass rates are due to surface defects in the pseudo-CT studies. This statement may not be valid when bone is included in the irradiation volume, as for instance in a rectal cancer case where the tumour bed or the high-risk area is very close to the sacrum. 

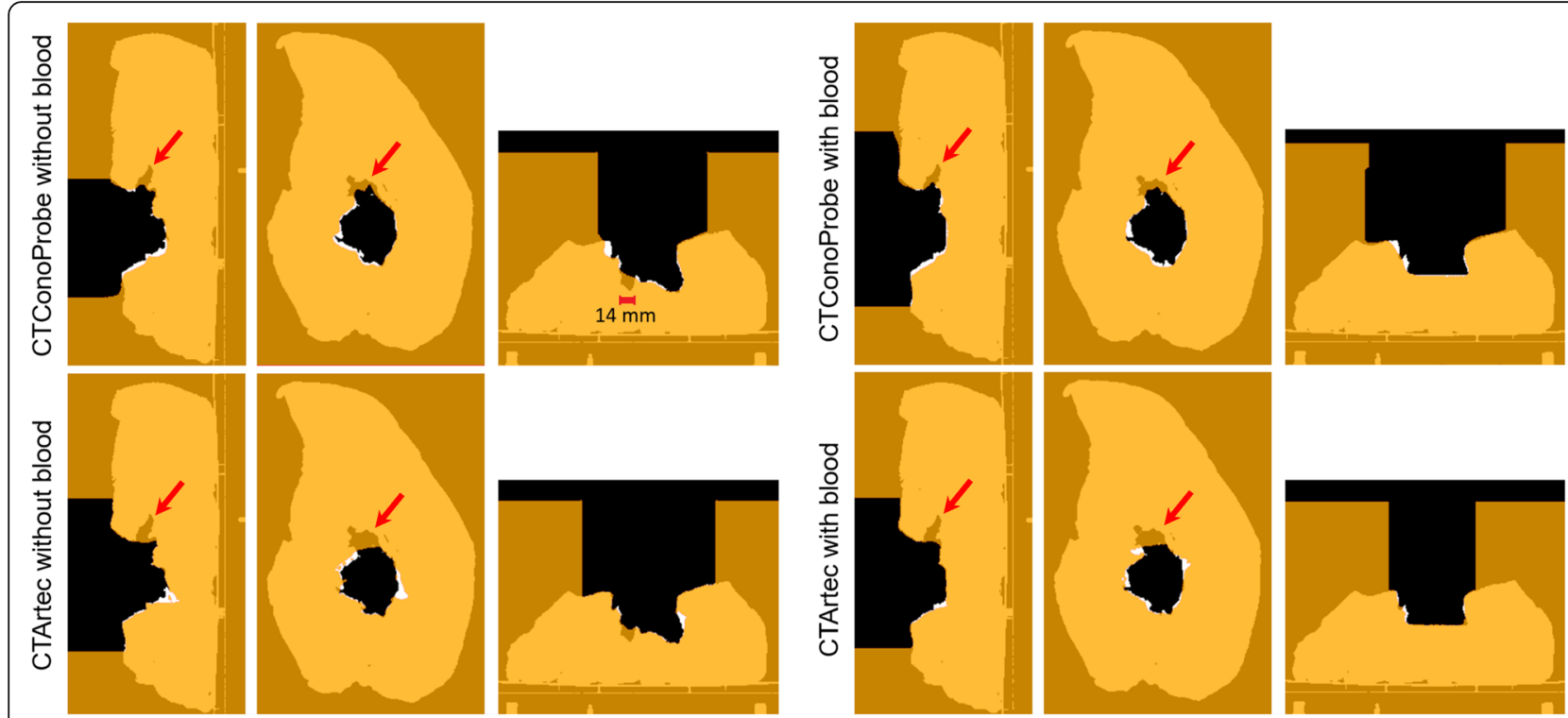

Fig. 3 Comparison between CTtissue\&air and the pseudo-CT studies CTConoProbe and CTArtec in both configurations (with and without blood). In this figure, each pseudo-CT study (tissue depicted in orange colour) was superimposed on CTtissue\&air (tissue represented in white colour). In all cases, air was depicted in black colour. Therefore, tissue in the pseudo-CT study matches that in CTtissue\&air when orange and white colours overlap. On the other hand, air in the pseudo-CT study matches that in CTtissue\&air when black colour is shown in the figure. Other options represent mismatches. From left to right in each configuration, sagittal, coronal and axial views. The red arrows point to a deep hole not completely scanned

In the simulated IOERT scenario, the conventional assumption of water-equivalent tissues at the applicator end led to inaccurate dose distributions in both configurations (average gamma pass rate $39.9 \%$ for dose values greater than $10 \%)$. The configuration with blood presented a different surface of the simulated tumour bed (irradiation surface flatter than that without blood) and a different air gap compared with the configuration without blood. Results improved when surfaces were considered in the dose calculation although there were regions in the surgical field that did not completely match, such as the 14-mm-width hole in the configuration without blood (Fig. 3, axial view of CTConoProbe and CTArtec). This hole could not be properly scanned since a small piece of tissue protruded and covered part of the hole. On the other hand, both devices could scan a surgical field with blood.

The match between CTtissue\&air and the pseudo-CT studies CTConoProbe and CTArtec in the target volume was better in the case of Artec compared with ConoProbe (Fig. 3). Similarly, gamma pass rates with Artec were better than those with ConoProbe (average values $92.9 \%$ and $88.5 \%$ for dose values greater than $10 \%$

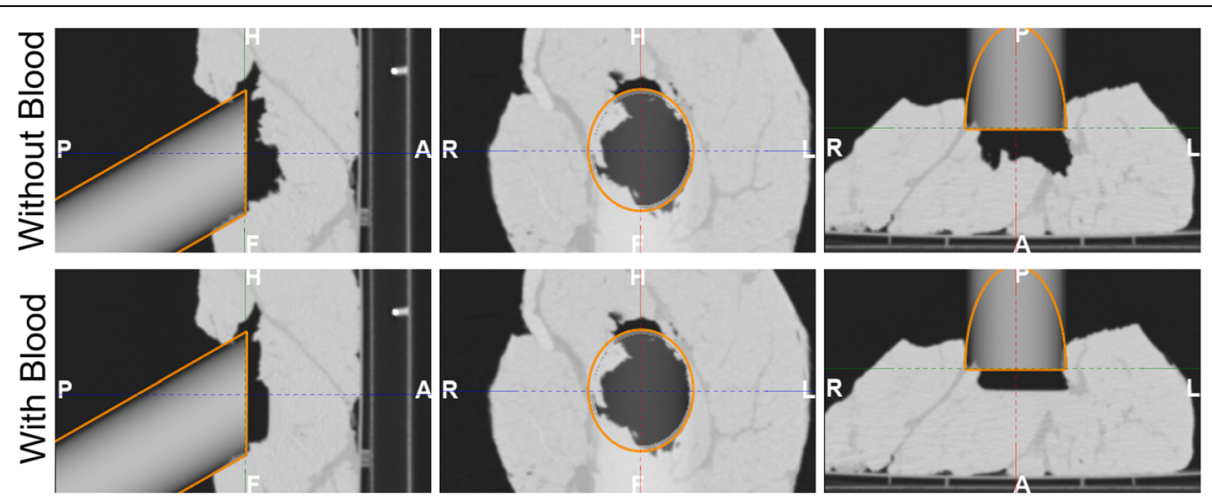

Fig. 4 IOERT scenario in both configurations (with and without blood). Virtual applicator (grey cylinder with its contour in orange colour) superimposed on CTpreSurf. This figure shows the surface irregularities of the irradiated volume and the air gap from the applicator end to the simulated tumour bed. From left to right, sagittal, coronal and axial views. H (head), F (feet), A (anterior), P (posterior), R (right) and L (left) 


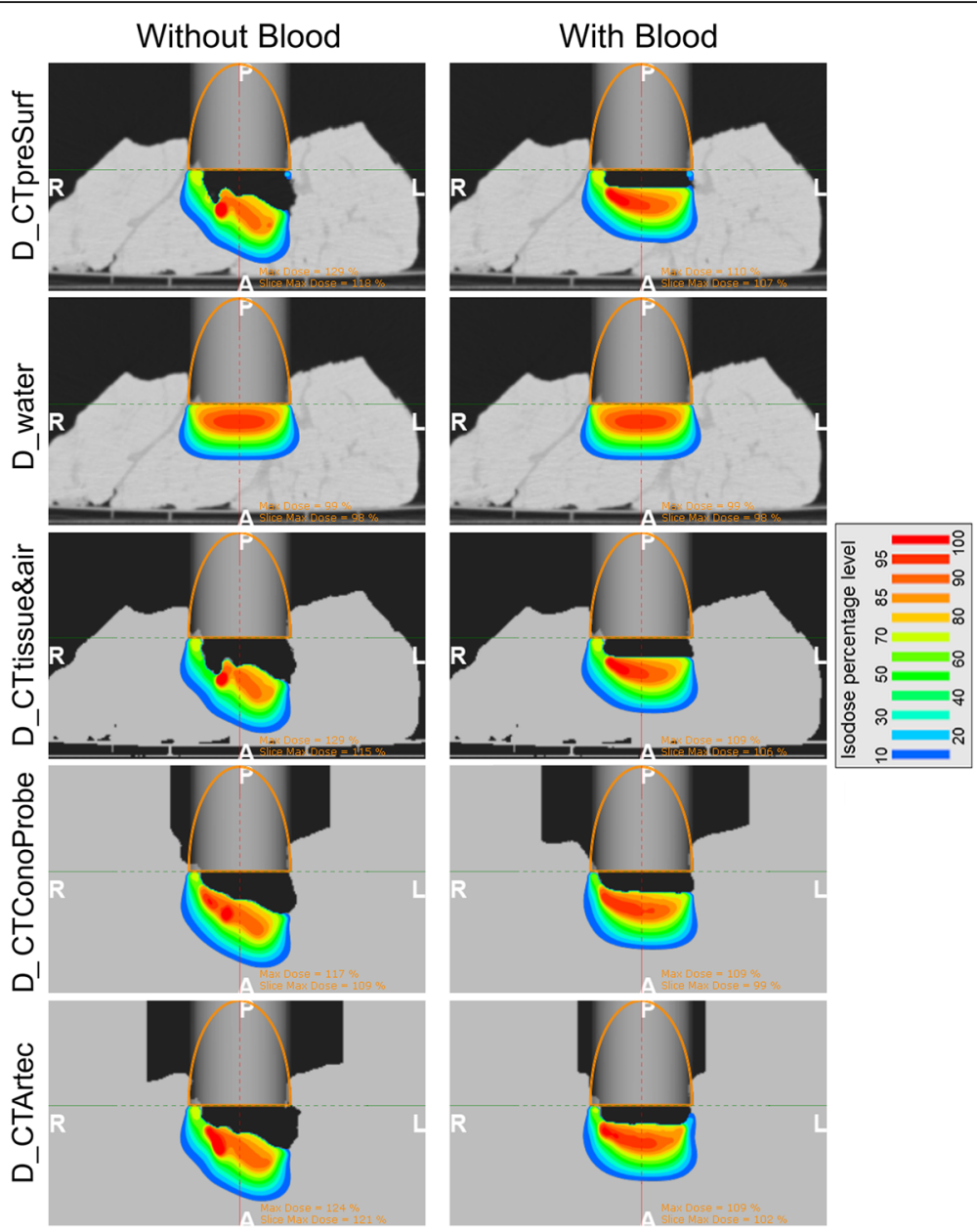

Fig. 5 3D dose distributions calculated using the Monte Carlo algorithm (axial view at the bevel centre). Virtual applicator represented as a grey cylinder with its contour in orange colour. H (head), F (feet), A (anterior), P (posterior), R (right) and L (left)

respectively). Possible sources of error in the ConoProbe measurements were the surface reconstruction from noisy points (PSE around $2 \mathrm{~mm}$, Table 2) and larger error in the location of the markers compared with Artec (fiducial registration error [FRE] Table 2).
The pseudo-CT study CTreproSurf fitted the CT study used to generate reproSurf (namely CTtissue\&air). These CT studies were utilised to assess the influence of the conversion from a $3 \mathrm{D}$ surface into a pseudo-CT study on the dose calculation. The results showed that the influence

Table 3 Percentage of voxels fulfilling the 3D gamma criteria ${ }^{a}$

\begin{tabular}{|c|c|c|c|}
\hline & & \multicolumn{2}{|l|}{$3 \% / 3 \mathrm{~mm}$} \\
\hline & & Dose $>10 \%$ & Dose $>70 \%$ \\
\hline \multirow[t]{4}{*}{ Without blood } & D_CTpreSurf v. D_water & 36.5 & 28.0 \\
\hline & D_CTpresurf v. D_CTtissue\&air & 99.6 & 99.8 \\
\hline & D_CTpreSurf v. D_CTConoProbe & 91.4 & 84.3 \\
\hline & D_CTpreSurf v. D_CTArtec & 93.6 & 88.2 \\
\hline \multirow[t]{4}{*}{ With blood } & D_CTpreSurf v. D_water & 43.2 & 31.9 \\
\hline & D_CTpreSurf v. D_CTtissue\&air & 99.8 & 99.9 \\
\hline & D_CTpreSurf v. D_CTConoProbe & 85.6 & 85.9 \\
\hline & D_CTpreSurf v. D_CTArtec & 92.1 & 90.9 \\
\hline
\end{tabular}

a Dose matrices: $151 \times 298 \times 320$. Voxel size $1.0 \times 1.0 \times 1.0 \mathrm{~mm}$. Gamma pass rates $\geq 90 \%$ are highlighted in bold

Average gamma pass rates for dose values greater than $10 \%: 39.9 \%, 99.7 \%, 88.5 \%$ and $92.9 \%$ for D_water, D_CTtissue\&air, D_CTConoProbe and D_CTArtec respectively 


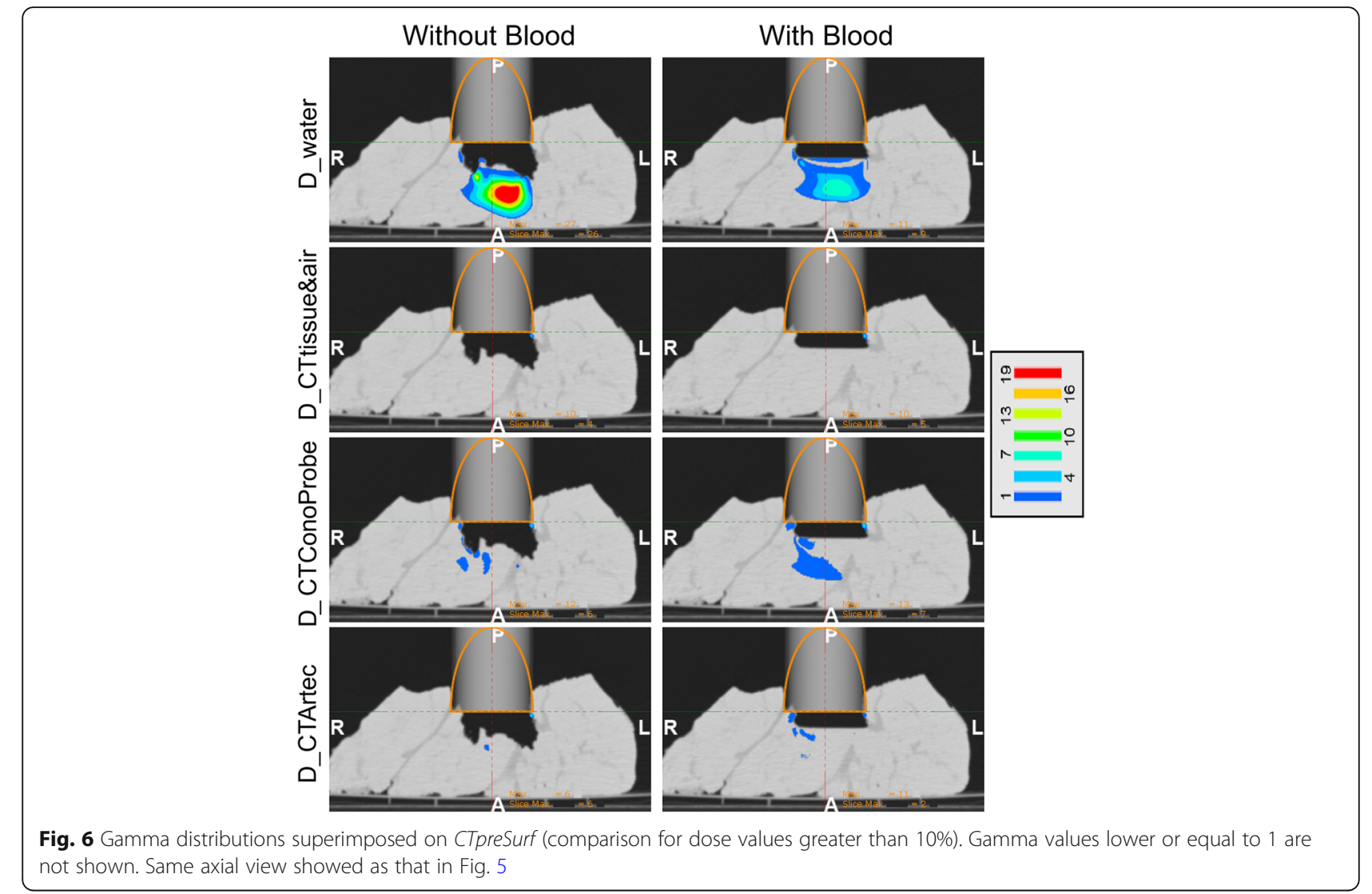

was negligible for the configuration without blood (the worst case of those presented in this study). Therefore, the error in the dose distributions D_CTConoProbe and D_CTArtec was caused by the inaccuracies of the scanned surfaces, and the registration between the 3D surfaces and CTpreSurf. 3D scanning from multiple viewpoints could improve the acquired data and thus increase the gamma pass rates, although it would further alter the surgical workflow.

Other studies have proposed in-vivo dosimetry with micro metal oxide semiconductor field-effect transistors, radiochromic films or thermoluminescence radiation detectors, which enables point and 2D dose measurements in IOERT $[8,36,37]$. Our approach focuses only on
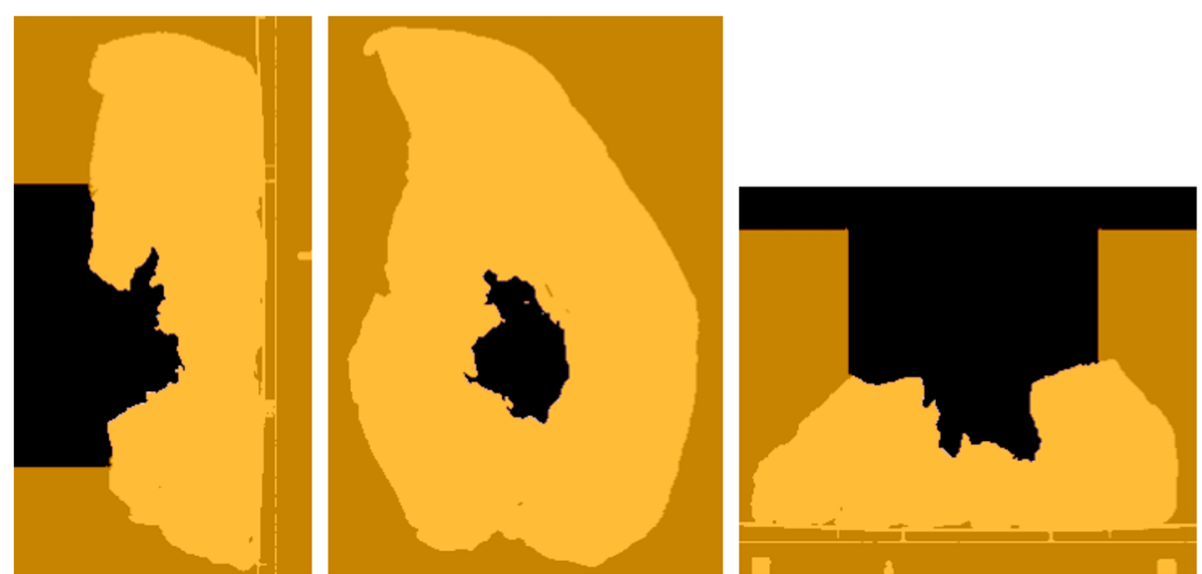

Fig. 7 Comparison between CTtissue\&air and the pseudo-CT study CTreproSurf. CTreproSurf (tissue depicted in orange colour) was superimposed on CTtissue\&air (tissue represented in white colour). In both cases, air was depicted in black colour. More information on how to interpret the figure colours in Fig. 3 
obtaining 3D dose distributions and some issues could still be improved. Applicator position and angle of beam incidence (applicator rotation) in relation to the patient's anatomy are required for dose calculations. After scanning the surface of the tumour bed, the applicator position and rotation could be collected with an optical tracking system [38], and then combined with that scanned surface to also obtain the air gap from the applicator end to the surface of the irradiated volume. The applicator data would be in the same coordinate space as the scanned surface when using ConoProbe (same optical tracking system) while a calibration tool visible to the tracking system and the Artec scanner should be added to the scenario to map the applicator pose to the scanned surface. Finally, it would be necessary to reduce the data post-processing steps detailed in section "Surface to pseudo-CT study for IOERT dose calculation" and section "Results" in order to obtain a real-time implementation in the clinical practice, although this is not crucial since the main interest was to obtain the actual dose distribution for quality assurance of IOERT, but not immediately before irradiation.

\section{Conclusions}

This is the first study that explores the use of surface scanning for 3D dose calculation in IOERT. In the simulated IOERT scenario, the conventional assumption of water-equivalent tissues at the applicator end led to inaccurate dose distributions. More accurate dose distributions were obtained when considering surfaces in the dose calculation of our simulated surgical field. The structured-light 3D scanner provided the best results in terms of dose distributions. Surface scanning is a promising method that could be easily included in the clinical practice, since the acquisition process is simple and does not require much time, and the calculated dose is comparable to the one that could be obtained with CT imaging. The findings obtained in this specific experimental setup warrant further research on surface scanning in the IOERT context owing to the clinical interest of improving the documentation of the actual IOERT scenario.

\footnotetext{
Abbreviations

2D: Two-dimensional; 3D: Three-dimensional; CT: Computed tomography; CTArtec: Pseudo-CT study obtained from Artec surface;

CTConoProbe: Pseudo-CT study obtained from ConoProbe surface; CTpostSurf: CT study of the surgical field and the markers acquired after surface scanning; CTpreSurf: CT study of the surgical field and the markers acquired before surface scanning; CTreproSurf: Pseudo-CT study obtained from reproSurf; $C T$ tissue\&air: Pseudo-CT study obtained after converting CTpreSurf data to only two values, tissue and air; D_CTArtec: 3D dose distribution calculated from CTArtec; D_CTConoProbe: 3D dose distribution calculated from CTConoProbe; D_CTpreSurf: 3D dose distribution calculated from CTpreSurf; D_CTreproSurf: 3D dose distribution calculated from CTreproSurf; D_CTtissue\&air: 3D dose distribution calculated from CTtissue\&air; D water: 3D dose distribution calculated under the conventional assumption of water-equivalent tissues at the applicator end;
}

FRE: Fiducial registration error; HU: Hounsfield units; IOERT: Intraoperative electron radiation therapy; LINAC: Linear accelerator; PSE: Point-surface error; reproSurf: Surface generated from CTtissue\&air; RMSE: Root-mean-square error; TPS: Treatment planning system; TRE: Target registration error

\section{Acknowledgements}

The authors extend their gratitude to GMV SA (Madrid, Spain), Alexandra de Francisco López, Yolanda Sierra Palomares, and medical physicists and radiotherapy technicians at Hospital General Universitario Gregorio Marañón (Madrid, Spain) for their valuable technical support.

\section{Funding}

This study was supported by Ministerio de Ciencia, Innovación y Universidades [grant number TEC2013-48251-C2-1-R]; by Ministerio de Ciencia, Innovación y Universidades, Instituto de Salud Carlos III and European Regional Development Fund (FEDER) Funds from the European Commission, "A way of making Europe" [grant numbers DTS14/00192, PI15/ 02121]; and by Comunidad de Madrid [grant number TOPUS-CM S2013/MIT3024]. The CNIC is supported by the Ministerio de Ciencia, Innovación y Universidades and the Pro CNIC Foundation, and is a Severo Ochoa Center of Excellence (SEV-2015-0505).

Study sponsors had no involvement in the study design, in the collection, analysis and interpretation of data; in the writing of the manuscript; and in the decision to submit the manuscript for publication.

\section{Availability of data and materials}

The datasets used and/or analysed during the current study are available from the corresponding author on reasonable request.

\section{Authors' contributions}

VGV, BSL, FAC and JP contributed in the study design. VGV and BSL participated in data collection. VGV and BSL contributed to data analysis. VGV, BSL, FAC, JJV, MD and JP provided data interpretation. VGV wrote the draft manuscript. VGV, BSL, FAC, JJV, MD and JP revised the manuscript. JP supervised this study. All authors read and approved the final manuscript.

\section{Ethics approval and consent to participate}

Not applicable.

\section{Consent for publication}

Not applicable.

\section{Competing interests}

The authors declare that they have no competing interests.

\section{Publisher's Note}

Springer Nature remains neutral with regard to jurisdictional claims in published maps and institutional affiliations.

\section{Author details}

${ }^{1}$ Instituto de Investigación Sanitaria Gregorio Marañón. Calle Doctor Esquerdo, 46, 28007, Madrid, Spain. '2Departamento de Oncología, Hospital General Universitario Gregorio Marañón, Madrid, Spain. ${ }^{3}$ Facultad de Medicina, Universidad Complutense de Madrid, Madrid, Spain. ${ }^{4}$ Clínica Universidad de Navarra, Madrid, Spain. ${ }^{5}$ Departamento de Bioingeniería e Ingeniería Aeroespacial, Universidad Carlos III de Madrid, Madrid, Spain. ${ }^{6}$ Centro de Investigación Biomédica en Red de Salud Mental (CIBERSAM), Madrid, Spain. ${ }^{7}$ Centro Nacional de Investigaciones Cardiovasculares Carlos III (CNIC), Madrid, Spain.

Received: 13 July 2018 Accepted: 12 November 2018 Published online: 07 December 2018

\section{References}

1. Calvo FA, Meirino RM, Orecchia R. Intraoperative radiation therapy - First part: rationale and techniques. Crit Rev Oncol Hematol. 2006;59:106-15.

2. Nag S, Willett CG, Gunderson LL, Harrison LB, Calvo FA, Biggs P. IORT with electron-beam, high-dose-rate brachytherapy or low-kV/electronic brachytherapy: methodological comparisons. In: Gunderson LL, Willett CG, Calvo FA, Harrison LB, editors. Intraoperative irradiation: techniques and results. New York: Humana Press; 2011. p. 99-115. 
3. Calvo FA. Intraoperative irradiation: precision medicine for quality cancer control promotion. Radiat Oncol. 2017;12:36.

4. Biggs P, Willett CG, Rutten H, Ciocca M, Gunderson LL, Calvo FA. Intraoperative electron beam irradiation: physics and techniques. In: Gunderson LL, Willett CG, Calvo FA, Harrison LB, editors. Intraoperative irradiation: techniques and results. New York: Humana Press; 2011. p. 51-72.

5. Valentini V, Balducci M, Tortoreto F, Morganti AG, De Giorgi U, Fiorentini G. Intraoperative radiotherapy: current thinking. Eur J Surg Oncol. 2002;28:180-5.

6. Ciocca M, Orecchia R, Garibaldi C, Rondi E, Luini A, Gatti G, et al. In vivo dosimetry using radiochromic films during intraoperative electron beam radiation therapy in early-stage breast cancer. Radiother Oncol. 2003;69:285-9.

7. Petoukhova A, Rüssel I, Nijst-Brouwers J, van Wingerden K, van Egmond J, Jacobs D, et al. In vivo dosimetry with MOSFETs and GAFCHROMIC films during electron IORT for accelerated partial breast irradiation. Phys Med. 2017:44:26-33.

8. Costa F, Sarmento S, Gomes D, Magalhães H, Arrais R, Moreira G, et al. In vivo dosimetry using Gafchromic films during pelvic intraoperative electron radiation therapy (IOERT). Br J Radiol. 2016;89:20160193.

9. Costa F, Sarmento S, Sousa O. Assessment of clinically relevant dose distributions in pelvic IOERT using Gafchromic EBT3 films. Phys Med. 2015; 31:692-701.

10. García-Vázquez V, Marinetto E, Guerra P, Valdivieso-Casique MF, Calvo FÁ, Alvarado-Vásquez $\mathrm{E}$, et al. Assessment of intraoperative 3D imaging alternatives for IOERT dose estimation. Z Med Phys. 2017;27:218-31.

11. Hensley FW. Present state and issues in IORT physics. Radiat Oncol. 2017; 12:37.

12. Portalés C, Gimeno J, Vera L, Fernández M. Towards a guidance system to aid in the dosimetry calculation of intraoperative electron radiation therapy. J Imaging. 2015;1:180-92.

13. Brudfors M, García-Vázquez V, Sesé-Lucio B, Marinetto E, Desco M, Pascau J. ConoSurf: open-source 3D scanning system based on a conoscopic holography device for acquiring surgical surfaces. Int J Med Robot. 2017;13:e1788.

14. Burgner J, Simpson AL, Fitzpatrick JM, Lathrop RA, Herrell SD, Miga Ml, et al. A study on the theoretical and practical accuracy of conoscopic holography-based surface measurements: toward image registration in minimally invasive surgery. Int J Med Robot. 2013;9:190-203.

15. Simpson AL, Burgner J, Glisson CL, Herrell SD, Ma B, Pheiffer TS, et al. Comparison study of intraoperative surface acquisition methods for surgical navigation. IEEE Trans Biomed Eng. 2013;60:1090-9.

16. Simpson AL, Sun K, Pheiffer TS, Rucker DC, Sills AK, Thompson RC, et al. Evaluation of conoscopic holography for estimating tumor resection cavities in model-based image-guided neurosurgery. IEEE Trans Biomed Eng. 2014; 61:1833-43.

17. Álvarez I, Enguita JM, Frade M, Marina J, Ojea G. On-line metrology with conoscopic holography: beyond triangulation. Sensors (Basel). 2009;9:7021-37.

18. OEM Manual for OPTIMET'S Mark10/10HD (P/N 3J06009, Rev 2): Optimet Optical Metrology Ltd. http://www.optimet.com. Accessed 31 Nov 2017.

19. Kazhdan M, Bolitho M, Hoppe H. Poisson surface reconstruction. Symp Geom Process. 2006:61-70.

20. Cignoni P, Callieri M, Corsini M, Dellepiane M, Ganovelli F, Ranzuglia G. MeshLab: an open-source mesh processing tool. Sixth Eurographics Italian Chapter Conference. 2008:129-36.

21. Professional 3D scanning solutions (Artec scanners brochure, 001-05/2017ENG): Artec 3D. https://www.artec3d.com. Accessed 31 Nov 2017.

22. Pascau J, Santos Miranda JA, Calvo FA, Bouché A, Morillo V, González-San Segundo $C$, et al. An innovative tool for intraoperative electron beam radiotherapy simulation and planning: description and initial evaluation by radiation oncologists. Int J Radiat Oncol Biol Phys. 2012;83:e287-e95.

23. Valdivieso-Casique MF, Rodríguez R, Rodríguez-Bescós S, Lardíes D, Guerra P, Ledesma MJ, et al. RADIANCE - a planning software for intra-operative radiation therapy. Transl Cancer Res. 2015;4:196-209.

24. Herranz E, Herraiz JL, Ibáñez P, Pérez-Liva M, Puebla R, Cal-González J, et al, Phase space determination from measured dose data for intraoperative electron radiation therapy. Phys Med Biol. 2015;60:375-401.

25. Guerra P, Udías JM, Herranz E, Santos-Miranda JA, Herraiz JL, Valdivieso MF, et al. Feasibility assessment of the interactive use of a Monte Carlo algorithm in treatment planning for intraoperative electron radiation therapy. Phys Med Biol. 2014;59:7159-79.

26. Autodesk Meshmixer. http://www.meshmixer.com. Accessed 31 Nov 2017.
27. Aitkenhead AH. Mesh voxelisation. https://es.mathworks.com/matlabcentral/ fileexchange/27390-mesh-voxelisation. Accessed 1 Feb 2017.

28. Patil S, Ravi B. Voxel-based representation, display and thickness analysis of intricate shapes. Proceedings of the 9th international conference on computer aided design and. Computer Graphics. 2005.

29. Arun KS, Huang TS, Blostein SD. Least-squares fitting of two 3-D point sets. IEEE Trans Pattern Anal Mach Intell. 1987;9:698-700.

30. Fedorov A, Beichel R, Kalpathy-Cramer J, Finet J, Fillion-Robin J-C, Pujol S, et al. 3D slicer as an image computing platform for the quantitative imaging network. Magn Reson Imaging. 2012;30:1323-41.

31. Ungi T, Lasso A, Fichtinger G. Open-source platforms for navigated imageguided interventions. Med Image Anal. 2016;33:181-6.

32. Bresciani S, Di Dia A, Maggio A, Cutaia C, Miranti A, Infusino E, et al. Tomotherapy treatment plan quality assurance: the impact of applied criteria on passing rate in gamma index method. Med Phys. 2013:40:121711.

33. Phillips N. Berry \& Kohn's operating room technique. St. Louis: Elsevier Health Sciences; 2016

34. Soriani A, laccarino G, Felici G, Ciccotelli A, Pinnarò P, Giordano C, et al. Development and optimization of a beam shaper device for a mobile dedicated IOERT accelerator. Med Phys. 2012;39:6080-9.

35. Takanen S, Gambirasio A, Gritti G, Källi M, Andreoli S, Fortunato M, et al. Breast cancer electron intraoperative radiotherapy: assessment of preoperative selection factors from a retrospective analysis of 758 patients and review of literature. Breast Cancer Res Treat. 2017;165:261-71.

36. Galimberti V, Ciocca M, Leonardi MC, Zanagnolo V, Paola B, Manuela S, et al. Is electron beam intraoperative radiotherapy (ELIOT) safe in pregnant women with early breast cancer? In vivo dosimetry to assess fetal dose. Ann Surg Oncol. 2009;16:100-5.

37. López-Tarjuelo J, Bouché-Babiloni A, Morillo-Macías V, de Marco-Blancas N, Santos-Serra A, Quirós-Higueras JD, et al. In vivo dosimetry in intraoperative electron radiotherapy: microMOSFETs, radiochromic films and a generalpurpose linac. Strahlenther Onkol. 2014;190:1060-5.

38. García-Vázquez V, Marinetto E, Santos-Miranda JA, Calvo FA, Desco M, Pascau J. Feasibility of integrating a multi-camera optical tracking system in intra-operative electron radiation therapy scenarios. Phys Med Biol. 2013;58: 8769-82.

Ready to submit your research? Choose BMC and benefit from

- fast, convenient online submission

- thorough peer review by experienced researchers in your field

- rapid publication on acceptance

- support for research data, including large and complex data types

- gold Open Access which fosters wider collaboration and increased citations

- maximum visibility for your research: over $100 \mathrm{M}$ website views per year

At BMC, research is always in progress.

Learn more biomedcentral.com/submissions 